\title{
Heart Rate Variability Biofeedback Increased Autonomic Activation and Improved Symptoms of Depression and Insomnia among Patients with Major Depression Disorder
}

\author{
I-Mei Lin ${ }^{1,2, *}$, Sheng-Yu Fan ${ }^{3, *}$, Cheng-Fang Yen ${ }^{4,5}$, Yi-Chun Yeh ${ }^{4,5}$, Tze-Chun Tang ${ }^{4,6}$, Mei-Feng Huang ${ }^{4,5}$, \\ Tai-Ling Liu ${ }^{4,5}$, Peng-Wei Wang ${ }^{4,5}$, Huang-Chi Lin ${ }^{4,5}$, Hsin-Yi Tsai ${ }^{1,4}$, Yu-Che Tsai ${ }^{1}$ \\ ${ }^{1}$ Department of Psychology, College of Humanities and Social Sciences, Kaohsiung Medical University, Departments of ${ }^{2}$ Medical Research and \\ ${ }^{4}$ Psychiatry, Kaohsiung Medical University Hospital, Kaohsiung City, ${ }^{3}$ Institute of Gerontology, College of Medicine, National Cheng Kung \\ University, Tainan City, ${ }^{5}$ Graduate Institute of Medicine and Department of Psychiatry, School of Medicine, College of Medicine, Kaohsiung \\ Medical University, ${ }^{6}$ Dr. Tang's Psychiatric Clinic and Mind Center, Kaohsiung City, Taiwan
}

\begin{abstract}
Objective: Autonomic imbalance is considered a psychopathological mechanism underlying major depressive disorder (MDD). Heart rate variability (HRV) is an index for autonomic activation. Poor sleep quality is common among patients with MDD. HRV biofeedback (BF) has been used for regulating autonomic balance among patients with physical illness and mental disorders. The purpose of present study was to examine the effects of HRV-BF on depressive symptoms, sleep quality, pre-sleep arousal, and HRV indices, in patients with MDD and insomnia.

Methods: In this case-controlled study, patients with MDD and Pittsburgh Sleep Quality Index (PSQI) score higher than 6 were recruited. The HRV-BF group received weekly 60-minute protocol for 6 weeks, and the control group who have matched the age and sex received medical care only. All participants were assessed on Beck Depression Inventory-II, Back Anxiety Inventory, PSQI, and Pre-Sleep Arousal Scale. Breathing rates and electrocardiography were also performed under resting state at pre-testing, and post-testing conditions and for the HRV-BF group, also at 1-month follow-up.

Results: In the HRV-BF group, symptoms of depression and anxiety, sleep quality, and pre-sleep arousal were significantly improved, and increased HRV indices, compared with the control group. Moreover, in the HRV-BF group, significantly improved symptoms of depression and anxiety, decreased breathing rates, and increased HRV indices were detected at post-testing and at 1-month follow-up, compared with pre-testing values.

Conclusion: This study confirmed that HRV-BF is a useful psychosocial intervention for improving autonomic balance, baroreflex, and symptoms of depression and insomnia in MDD patients.
\end{abstract}

KEY WORDS: Heart rate variability biofeedback; Major depressive disorder; Insomnia.

\section{INTRODUCTION}

The World Health Organization estimates that depressive disorders are the third leading cause of disease burden, accounting for a $4.3 \%$ of the global burden of dis-

Received: April 11, 2018/ Revised: June 3, 2018

Accepted: June 15, 2018

Address for correspondence: Yu-Che Tsai, PhD

Department of Psychology, College of Humanities and Social

Sciences, Kaohsiung Medical University, 100 Shih-Chuan 1st

Road, Kaohsiung City 807, Taiwan

Tel: +886-73215422 ext. 838, Fax: +886-73233716

E-mail: psyyct@kmu.edu.tw

ORCID: https://orcid.org/0000-0002-5947-5823

*These authors contributed equally to this study as co-first authors. eases by 2030. ${ }^{1)}$ Insomnia and poor sleep quality are common in patients with the major depressive disorder (MDD). According to the Sequenced Treatment Alternatives to Relieve Depression report, $87.4 \%$ of MDD patients comorbid at least one insomnia symptom, such as sleep onset insomnia, mid-nocturnal insomnia, or early morning insomnia. Insomnia symptoms have been related to severe depressive symptoms, poor quality of life, and impaired psychological and physiological functions. $^{2)}$

Heart rate variability $(\mathrm{HRV})$ is a biological marker of the autonomic nervous system (ANS), with decreased HRV indices being associated with MDD patients and probably

(ㄷ) This is an Open-Access article distributed under the terms of the Creative Commons Attribution Non-Commercial License (http://creativecommons.org/licenses/by-nc/4.0) which permits unrestricted non-commercial use, distribution, and reproduction in any medium, provided the original work is properly cited. 
being a biomarker of depression. ${ }^{3)}$ Patients with MDD and comorbid insomnia may present with ANS or cortical hyperarousal, and demonstrate higher physical and cognitive arousal as clinical symptoms. ${ }^{4)}$ Low HRV indicates an imbalance between the sympathetic and parasympathetic nervous systems, or reduced baroreflex sensitivity. A meta-analysis of 18 studies demonstrated lower HRV (standard deviation of normal to normal intervals, SDNN; high-frequency power, HF) and higher low-frequency power/high-frequency power (LF/HF) ratio in MDD patients, when compared to healthy controls. Kemp et al. ${ }^{5-8)}$ conducted a series of studies exploring the associations between depression and HRV, as measured from a 2-minute electrocardiogram (ECG) at resting state. The results indicated low HRV in patients with MDD or MDD subtypes, such as MDD comorbid with anxiety disorder or generalized anxiety disorder. ${ }^{5-8)}$ Similar results were reported for older Brazilian patients with newly diagnosed treatment-naïve depression. ${ }^{9,10)}$ Wang et al. ${ }^{11)}$ matched age and sex between the patients with MDD and healthy controls, and observed lower HRV indices in patients with MDD than that in the healthy controls, including HF, SDNN, SDANN (average SDNN), root mean square successive difference (rMSSD), and the proportion derived by diving the number of successive normal to normal (NN) interval pairs that differ greater than $50 \mathrm{~ms}$ (NN50) by the total number of NN intervals (pNN50). The severity of depression was positively associated with heart rate (HR) and the LF/HF ratio of HRV, while negatively associated to SDNN and HF of HRV. ${ }^{6,11)}$

Previous studies have also demonstrated higher nocturnal HRs and lower HRV in patients with primary insomnia as compared to healthy controls. ${ }^{12)}$ Although Kemp et $a l^{5-8)}$ confirmed the association between depression and HRV, the 2-minute ECG measured in their studies may be too short to reflect ANS and baroreflex activations. Few studies have explored HRV focusing on insomnia symptoms in patients with MDD.

Antidepressant medication is frequently used to reduce symptoms of depression and insomnia and to recover mood, cognition, and physiological functions. However, $30 \%$ to $50 \%$ of the patients did not respond to antidepressants ${ }^{13)}$ and antidepressants did not improve HRV indices. ${ }^{6)}$ HRV biofeedback (HRV-BF) is a noninvasive psychological intervention used widely in various physical illnesses and mental disorders. ${ }^{14)}$ HRV-BF trains par- ticipants to breathe at a resonance frequency rate (approximately 6 breaths/minute) and this leads to an increased baroreflex and vagal activity, as well as an improved balance between the sympathetic and parasympathetic nervous systems. ${ }^{14,15)}$ Karavidas et al. ${ }^{16)}$ were the first to apply a 10-session HRV-BF protocol in 11 MDD patients, and to observe increased SDNN of HRV and decreased depression scores at session 4. However, the SDNN of HRV returned to baseline levels and was not maintained post-testing or at a longer follow-up. Siepmann et al. ${ }^{17)}$ applied a 6-session HRV-BF to 14 MDD patients to observe increased pNN50 of HRV and decreased HR, anxiety and depressive symptoms. ${ }^{17)}$ However, it should be noted that no comparative controls were used in these two studies. ${ }^{16,17)}$ Caldwell and Steffen ${ }^{18)}$ combined HRV-BF with psychotherapy and reported an increased SDNN and greatly reduced depressive symptoms in MDD patients, as compared to the MDD group who received usual care treatment and non-MDD controls. To our knowledge, there is a lack of evidence on the effect of HRV-BF on patients with MDD and insomnia, and on their HRV indices at each HRV-BF training session.

Therefore, the purpose of the present study was to examine the effect of HRV-BF in patients who present with MDD and insomnia. We hypothesized that HRV-BF would lead to an increase in HRV indices and reduce symptoms of depression and insomnia.

\section{METHODS}

\section{Participants}

Patients with MDD were referred by psychiatrists from three hospitals. Inclusion criteria were as follows: (1) primary diagnosis of MDD based on the Diagnostic and Statistical Manual of Mental Disorders, 5th edition (DSM-5). Comorbid MDD and anxiety disorders were permitted as long as the primary diagnosis was $\mathrm{MDD}^{19)}$; (2) a score of Pittsburgh Sleep Quality Index (PSQI) higher than $6^{20)}$; and (3) patients' age were between 20 and 75 years. Exclusion criteria were as follows: (1) diagnosis of mental disorders other than depressive or anxiety disorders; report of current substance abuse, and cognitive impairment that would significantly affect the ability to learn and practice HRV-BF; and (2) depressive disorder due to another medical condition (e.g., cancer, kidney 
disease, stroke etc.).

Institutional review board's approval was obtained from the ethics committee of Kaohsiung Medical University Hospital, Taiwan (KMUH-IRB-20120209, KMUHIRB-F(I)-20160027) and Kaohsiung Chang Gung Memorial Hospital, Taiwan (CGMH IRB: 1604250002). Informed consent was obtained from each participant. Participants were compensated with New Taiwan dollar 300 (about USD 11) per session and received a $C D$ album for their participation.

\section{Psychological Questionnaires}

\section{Beck Depression Inventory-II}

The Beck Depression Inventory-II (BDI-II) includes 21 items scored on a 4-point Likert scale to assess depressive symptoms. ${ }^{21)}$ The total score of BDI-II ranges from 0 to 63 and yields two factors; cognitive and somatic depression. ${ }^{21)}$ The Cronbach $\alpha$ for the Chinese version of BDI-II was 0.94 , and split-half reliability was $0.91 .^{21)}$ The BDI-II total score was significantly related to the State-Trait Anxiety Inventory-Depression and State-Trait Anxiety Inventory-Anxiety $(r=0.76, p<0.001$; and $r=0.69, p<$ 0.001 , respectively). ${ }^{22)}$

\section{Beck Anxiety Inventory}

The Beck Anxiety Inventory (BAI) includes 21 items scored on a 4-point Likert scale to assess anxiety symptoms. The total score ranges from 0 to $0.63 .^{23)}$ The Cronbach $\alpha$ of the Chinese version of BAI was 0.95 , and split-half reliability was $0.91 .^{24)}$

\section{Pittsburgh Sleep Quality Index}

The PSQI includes a 19-item questionnaire that measures the sleep quality of the previous month, which is calculated by a seven-component score from: subjective sleep quality (C1), sleep latency (C2), sleep duration (C3), habitual sleep efficiency (C4), sleep disturbance (C5), use of sleeping medication (C6), and daytime dysfunction (C7). The total component score ranges from 0 to 21 and provides an index of sleep quality. Patients with a PSQI total score higher than six are considered as bad sleepers. ${ }^{20)}$ The Chinese version of PSQI has good psychometric properties and has been used extensively for sleep quality evaluation in Taiwanese patients. The Cronbach $\alpha$ was between 0.82 and 0.83 , and test - retest reliability of
14-21 days was 0.85 . The total score of PSQI was significantly different between insomnia group and healthy control group. ${ }^{20)}$

The raw scores of sleep efficiency, total time spent asleep, and total sleep time were also analyzed and calculated in this study. Sleep efficiency was defined as the ratio of total sleep time to time spent in bed (percentage).

\section{Pre-Sleep Arousal Scale}

The Pre-Sleep Arousal Scale (PSAS) includes 18 items scored on a 4-point Likert scale to assess pre-sleep arousal. PSAS includes two sub-scales: physical and cognitive arousals. ${ }^{25)}$ The Cronbach $\alpha$ of the Chinese version of PSAS was 0.88. There were significant associations between BAI total score and PSAS total score, physical and cognitive arousals $(r s=0.55,0.58$, and $0.41 ; p<0.001){ }^{26)}$

\section{Breathing Rates and Electrocardiogram Measurement}

A respiration belt with a stretch sensor was used to detect participants' chest expansion and contraction, measured for 5 minutes at resting baseline by using the ProComp Infiniti ${ }^{\mathrm{TM}}$ (Thought Technology Ltd., Montréal, QU, Canada). The sampling rate of respiration was 256 $\mathrm{Hz} / \mathrm{sec}$ and the raw signals from the respiration waveform were outputted for the calculation of the breathing rates.

Lead II ECG raw signals were collected by using the ProComp Infiniti ${ }^{\mathrm{TM}}$ with a sampling rate of $2,048 \mathrm{~Hz} / \mathrm{sec}$. ECGs were recorded for 5 minutes at baseline during pre-testing and post-testing for both HRV-BF and control groups, while the HRV-BF group received an ECG measurement also at 1-month follow-up. In addition, 5-minute ECGs were performed for the HRV-BF group at pre-training and post-training of 6 sessions. The CardioPro Infiniti HRV analysis module (Thought Technology) was used to detect and analyze the ECG raw signals including (1) acquire real-time interbeat intervals (IBIs) from ECG raw signals, and IBIs differing more than 20\% from the previous $\mathrm{IBI}$ were excluded by automatic filtering. If the frequency of ECG artifacts, detected by visual inspection in a 5 seconds window, was more than $5 \%$ of the total number of signals in the same period, data were deleted. Data were modified by adding, splitting, and averaging pairs of consecutive IBIs, and then calculated the time domain of the HRV index that includes SDNN. ${ }^{27)}$ Moreover, Fast Fourier transformation was used to transfer ECG raw signals to the frequency domain of HRV indices, including LF (0.04- 
$0.15 \mathrm{~Hz}), \mathrm{HF}(0.15-0.4 \mathrm{~Hz})$, and the LF/HF ratio.

\section{Study Design and Protocol}

In this case-control study, patients with MDD were recruited for HRV-BF training (the HRV-BF group); and the control group who were matched based on 5-year age difference and same sex. All MDD patients underwent the same procedures at pre-testing and post-testing conditions. Regarding the breathing rates and ECGs at pre-testing and post-testing, all participants were instructed to take their medications as usual, refrain from caffeinated and alcoholic beverages, as well as smoking, and to not exercise excessively three hours before the ECG recordings. In consideration of the circadian rhythm, ECGs were performed between 9 AM to 5 PM. Participants completed the psychological questionnaires and then received a 5-minute respiration and ECG recording at pre-testing and post-testing in a sound-attenuated and temperature-controlled room. Participants in the HRV-BF group received another respiration and ECG recording at 1-month follow-up.

Participants in the HRV-BF group received standard medical care and a weekly 60-minute HRV-BF for six weeks, and underwent post-testing and 1-month follow-up. The HRV-BF protocol was based on Lin et al. ${ }^{27)}$ who modified the HRV-BF protocol of Lehrer et al. ${ }^{15)}$ The HRV-BF protocol focused on autonomic balance through slow breathing at resonance frequency about 5.5 to 6 breaths/minute, and the contents of HRV-BF training includes self-guided muscle relaxation, diaphragmatic breathing, paced breathing, pursed lip breathing, psychoeducation of depression regulation, and a 10-minute daily homework.

Participants in the control group received standard medical care only, and then a post-testing measurement after six weeks. Participants in the control group were invited to receive HRV-BF training after post-testing, but these data were not merged into the HRV-BF group.

\section{Statistical Analysis}

Group differences in demographic data, medications, psychological questionnaires, breathing rates, HRV indices were examined by Student's $t$ test or chi-square. The effects of HRV-BF were examined as follow: (1) Two-way (2 groups $\times 2$ tests) repeated-measures analysis of variance (ANOVA) was used to examine group differ- ences on psychological questionnaires, breathing rates, and HRV indices at pre-testing and post-testing; (2) one-way repeated-measures ANOVA was used to examine the between-session effects on psychological questionnaires, breathing rates, and HRV indices at pre-testing, post-testing, and 1-month follow-up in the HRV-BF group alone. Mauchly sphericity test with GreenhouseGeisser adjustment and Bonferroni's post-hoc comparisons were applied in two-way and one-way repeated-measures ANOVA; (3) paired Student's $t$ tests were used for the evaluation of the within-session effects on HRV indices between pre-training and post-training in the HRV-BF group.

The effect size of partial eta squared $\left(\eta_{p}{ }^{2}\right)$ was also calculated. A $\eta_{\mathrm{p}}{ }^{2}$ lower than 0.05 represents a small effect size, between 0.06 and 0.14 reflects a medium effect size, and higher than 0.15 a large effect. ${ }^{28)}$ All analyses were performed using IBM SPSS predictive analytics software version 21.0 (IBM Corp., Armonk, NY, USA).

\section{RESULTS}

Forty-eight patients with MDD were age- and sex-matched between the HRV-BF (mean age, $38.50 \pm 11.66$ years) and control groups (mean age, $37.92 \pm 14.01$ years). There were 24 participants with 14 females and 10 males in each group. No significant differences between the two groups on demographic data, medications (including antidepressants, benzodiazepine, or hypnotics/sedatives), psychological questionnaires (including BDI-II, BAI, PSQI, and PSAS), breathing rates, or HRV indices were observed at the pre-testing (Table 1).

\section{Effects of HRV-BF on Depression, Anxiety, Sleep Quality, and Pre-sleep Arousal}

There were significant groupxtime interaction effects with a medium to large effect size on total score of BDI-II, cognitive depression, somatic depression, total score of BAI, total score of PSQI, total score of PSAS, and cognitive arousal of PSAS $(F=8.10, p=0.007 ; F=5.97, p=0.018$; $F=6.69, p=0.011 ; F=11.89, p=0.001 ; F=6.81, p=0.012$; $F=7.56, p=0.009$; and $F=10.98, p=0.002$, respectively). Bonferroni's post-hoc comparison indicated that the HRV-BF group had lower total score of BDI-II, cognitive depression, somatic depression, total score of BAI, total score of PSQI, total score of PSAS, and cognitive arousal 
Table 1. Demographic data and research variables between the HRV-BF and control groups

\begin{tabular}{|c|c|c|c|c|}
\hline Variable & HRV-BF group $(n=24)$ & Control group $(n=24)$ & $t \chi^{2}$ & $p$ \\
\hline Age (yr) & $38.50 \pm 11.66$ & $37.92 \pm 14.01$ & $\mathrm{t}=-0.16$ & 0.88 \\
\hline \multicolumn{5}{|l|}{ Sex } \\
\hline Female & $14(58.3)$ & $14(58.3)$ & & \\
\hline Male & $10(41.7)$ & $10(41.7)$ & $\chi^{2}=0.00$ & 1.00 \\
\hline \multicolumn{5}{|l|}{ Medications } \\
\hline Antidepressants & $18(75.00)$ & $20(83.33)$ & $\chi^{2}=0.772$ & 0.66 \\
\hline Benzodiazepine & $14(58.33)$ & $18(75.00)$ & $\chi^{2}=1.833$ & 0.31 \\
\hline Hypnotics/sedatives & $7(29.17)$ & $4(16.67)$ & $\chi^{2}=1.091$ & 0.49 \\
\hline Without medication or missing & $2(8.33)$ & $2(8.33)$ & $\chi^{2}=0.00$ & 1.00 \\
\hline \multicolumn{5}{|l|}{ BDI-II } \\
\hline Total score & $24.25 \pm 14.74$ & $24.92 \pm 10.62$ & $t=0.18$ & 0.86 \\
\hline Cognitive depression & $17.67 \pm 12.40$ & $18.92 \pm 8.78$ & $\mathrm{t}=0.4$ & 0.69 \\
\hline Somatic depression & $6.58 \pm 3.31$ & $6.00 \pm 2.36$ & $\mathrm{t}=-0.7$ & 0.49 \\
\hline BAI, total score & $20.96 \pm 9.45$ & $18.88 \pm 9.92$ & $\mathrm{t}=-0.75$ & 0.46 \\
\hline PSQI, total score & $12.42 \pm 3.20$ & $11.08 \pm 3.74$ & $\mathrm{t}=-1.33$ & 0.19 \\
\hline \multicolumn{5}{|l|}{ PSAS } \\
\hline Total score & $41.96 \pm 13.80$ & $39.33 \pm 11.61$ & $\mathrm{t}=-0.71$ & 0.48 \\
\hline Physical arousal & $16.58 \pm 6.01$ & $15.79 \pm 7.07$ & $\mathrm{t}=-0.42$ & 0.68 \\
\hline Cognitive arousal & $25.38 \pm 9.15$ & $23.96 \pm 7.56$ & $t=-0.59$ & 0.56 \\
\hline Breathing rate (breath/min) & $14.98 \pm 4.08$ & $14.90 \pm 3.53$ & $\mathrm{t}=-0.08$ & 0.94 \\
\hline HRV indices & $37.18 \pm 17.00$ & $35.60 \pm 16.06$ & $\mathrm{t}=-0.33$ & 0.74 \\
\hline \multicolumn{5}{|l|}{ SDNN (ms) } \\
\hline $\operatorname{LF}\left(\mathrm{ms}^{2}\right)$ & $151.64 \pm 218.22$ & $149.41 \pm 296.59$ & $t=-0.03$ & 0.98 \\
\hline $\mathrm{HF}\left(\mathrm{ms}^{2}\right)$ & $128.69 \pm 120.70$ & $130.97 \pm 134.90$ & $\mathrm{t}=0.06$ & 0.95 \\
\hline $\ln (\mathrm{LF})\left(\mathrm{ms}^{2}\right)$ & $4.25 \pm 1.36$ & $3.97 \pm 1.38$ & $\mathrm{t}=-0.70$ & 0.49 \\
\hline $\ln (\mathrm{HF})\left(\mathrm{ms}^{2}\right)$ & $4.27 \pm 1.33$ & $4.31 \pm 1.23$ & $\mathrm{t}=0.10$ & 0.92 \\
\hline $\mathrm{LF} / \mathrm{HF}$ ratio & $1.29 \pm 0.92$ & $1.12 \pm 1.17$ & $\mathrm{t}=-0.55$ & 0.59 \\
\hline Total power $\left(\mathrm{ms}^{2}\right)$ & $532.76 \pm 535.25$ & $483.90 \pm 469.09$ & $t=-0.34$ & 0.74 \\
\hline
\end{tabular}

Values are presented as mean \pm standard deviation or number (\%).

HRV, heart rate variability; BF, biofeedback; BDI-II, Beck Depression Inventory-II; BAl; Beck Anxiety Inventory; PSQI, Pittsburgh Sleep Quality Index; PSAS, Pre-Sleep Arousal Scale; SDNN, standard deviation of normal to normal intervals; LF, low frequency; HF, high frequency.

of PSAS at post-testing, as compared to the control group. Moreover, the HRV-BF group had lower total score of BDI-II, cognitive depression, somatic depression, total score of BAI, and cognitive arousal of PSAS at post-testing than the values at the pre-testing (Table 2 and Fig. 1). However, there was no significant group $x$ time interaction effect in physical arousal of PSAS ( $F=0.67, p=0.418)$.

\section{Effects of HRV-BF on Breathing Rates and HRV Indices}

There were significant groupxtime interaction effects with a medium to large effect on breathing rates $(F=$ 10.30, $p=0.002$ ) and HRV indices, including SDNN, LF, $\ln (\mathrm{LF}), \mathrm{LF} / \mathrm{HF}$ ratio and total power $(F=9.86, p=0.003$; $F=6.28, p=0.016 ; F=11.10, p=0.002 ; F=5.20, p=0.027$; and $F=8.05, p=0.007$, respectively). Bonferroni's post-hoc comparison indicated that the HRV-BF group had lower breathing rates and higher HRV indices (SDNN, In[LF], and LF/HF ratio) at post-testing, as compared to controls. Moreover, the HRV-BF group had lower breathing rates and higher HRV indices (SDNN, LF, In[LF], LF/HF ratio, and total power) at post-testing, as compare to the values at pre-testing. Breathing rates were significantly decreased from 14.98 (pre-testing) to 10.81 (post-testing) breaths/minute in the HRV-BF group; however, there was no significant change on breathing rates between pre-testing and post-testing in the control group (14.90 at pre-testing to 14.68 at post-testing breaths/minute). However, no significant groupxtime interaction effect on the HF or $\ln (\mathrm{HF})$ of the HRV was detected $(F=0.51, p=0.480$ and $F=1.50, p=0.226$, respectively) (Table 2 ).

\section{Effects of HRV-BF on Psychological Questionnaires, Breathing Rates and HRV Indices at Pre-test, Post-test, and 1-Month Follow-up}

In total, 19 participants in the HRV-BF group com- 


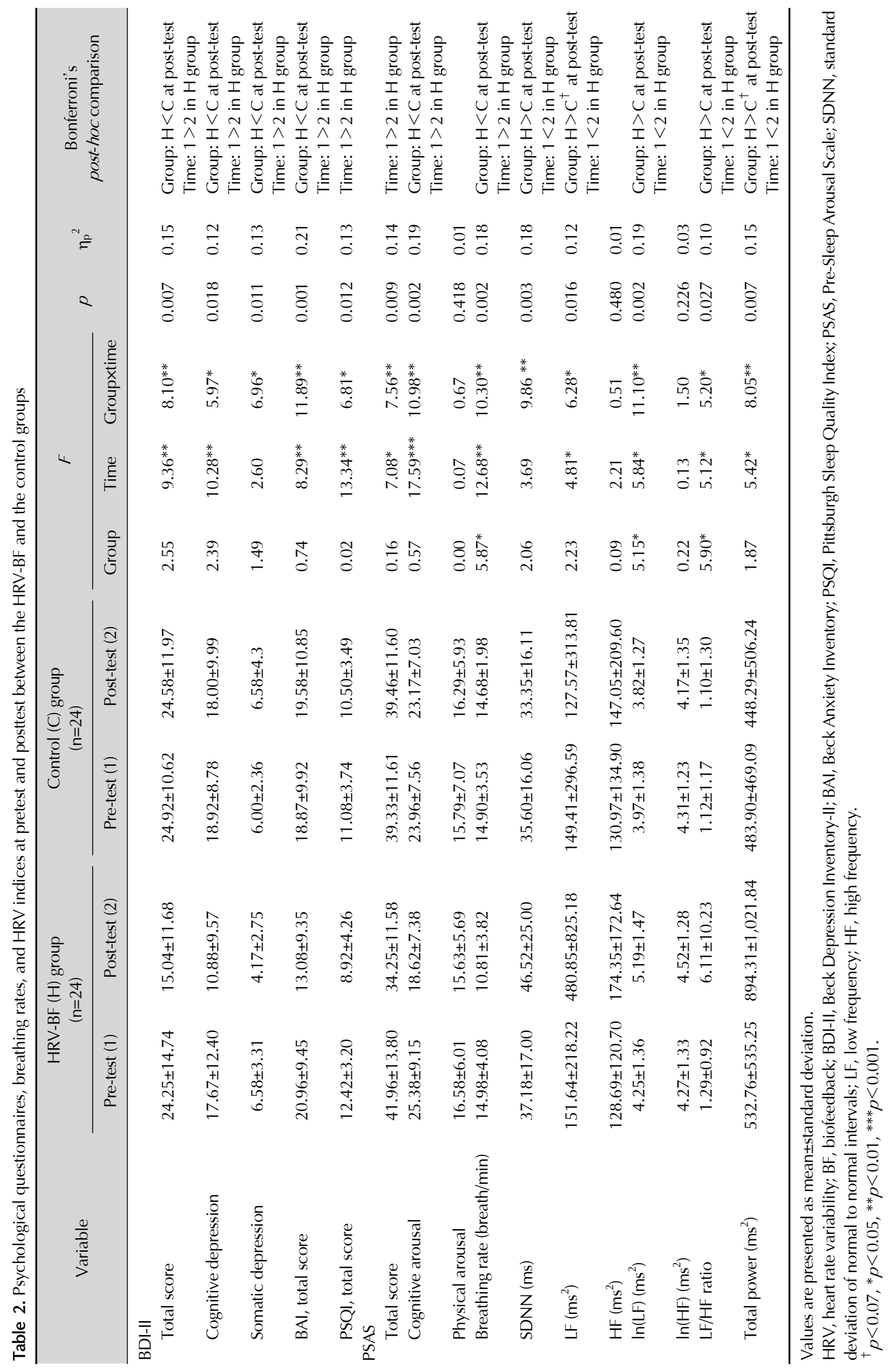




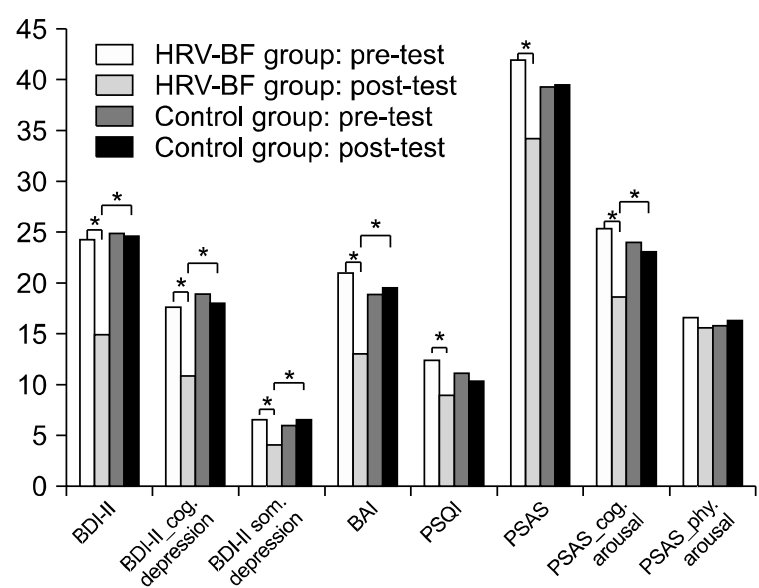

Fig. 1. The psychological questionnaires at pre- and post-tests for the heart rate variability biofeedback (HRV-BF) and control groups. BDI-II, Beck Depression Inventory-II; cog., cognitive; som., somatic; BAI, Beck Anxiety Inventory; PSQI, Pittsburgh Sleep Quality Index; PSAS, Pre-Sleep Arousal Scale; phy., physical. ${ }^{*} p<0.05$.

pleted pre-testing and post-testing, along with a 1-month follow-up measurement. However, one participant did not complete the questionnaires at the 1-month follow-up, and the participant's HRV data were damaged. Therefore, data from 18 participants were analyzed. After HRV-BF training, there was a significant decrease in total score of BDI-II score, cognitive depression, somatic depression, total score of BAI, PSQI, total score of PSAS, cognitive arousal, and physical arousal $(F=12.89, p<$ $0.001 ; F=12.85, p<0.001 ; F=7.46, p=0.002 ; F=12.32$, $p<0.001 ; F=7.99, p<0.001 ; F=8.35, p=0.001 ; F=7.92$, $p=0.002$; and $F=3.31, p<0.049$, respectively). Bonferroni's post-hoc comparison indicated that the HRV-BF group had lower total score of BDI-II, cognitive depression, and total score of BAI at post-testing and at 1-month follow-up, as compared to the values at pre-testing. Moreover, lower somatic depression, total score of PSQI, total score of PSAS, and cognition arousal were observed at post-testing compared to the values at pre-testing.

In addition, improved sleep quality in regards to subjective sleep quality (C1), sleep duration (C3), and daytime function (C7) were detected at post-test compared to the values at pre-test in the HRV-BF group $(F=5.55$, $p=0.008 ; F=3.32, p=0.048$; and $F=5.14, p=0.011$, respectively). The raw sleep efficiency score was significantly improved from $71.72 \%$ at pre-testing to $79.17 \%$ at post-testing, and $86.28 \%$ at 1 -month follow-up $(F=13.16, p<0.001)$. Patients with MDD in the HRV-BF group also presented with significantly reduced total time spent asleep (from 28.06 minutes at pre-testing to 22.22 minutes at post-testing) and increased total sleep time (from 5.58 hours at pre-testing to 6.64 hours at post-testing).

There was a significant decrease in breathing rate ( $F=6.44, p=0.004)$ at post-testing and at 1-month follow-up, as compared to pre-testing values in the HRV-BF group (14.56, 11.02, 11.07 breaths/minute, respectively). HRV indices, including SDNN and In(LF), in the HRV-BF group were also increased at post-test and at 1-month follow-up, as compared to pre-testing values $(F=6.57, p=$ 0.004 ; and $F=10.62, p<0.001$, respectively); Higher total power was also observed in the HRV-BF group at post-testing, as compared to pre-testing values ( $F=5.18$, $p=0.011$ ) (Table 3).

\section{Differences on HRV Indices between Pre-training and Post-training during HRV-BF Sessions}

There was significantly higher SDNN at post-training than that at pre-training for sessions 1 to $6(t=-3.17$, $p=0.004 ; t=-4.20, p<0.001 ; t=-5.16, p<0.001 ; t=$ $-2.51, p=0.020 ; t=-4.84, p<0.001$; and $t=-3.31, p=$ 0.003 , respectively); higher LF at post-training as compared to that at pre-training for sessions 1,3 , and 5 ( $t=$ -3.38, $p=0.003 ; t=-2.45, p=0.023$; and $t=-3.05, p=$ 0.006 , respectively); and higher total power at post-training than that at pre-training for sessions 2,3 , and $5(t=-2.72$, $p=0.012 ; t=-3.45, p=0.002$; and $t=-3.29, p=0.003$, respectively) (Fig. 2).

\section{DISCUSSION}

This study demonstrated that MDD patients comorbid with insomnia had significantly decreased symptoms of depression and anxiety, and improved insomnia and pre-sleep arousal after 6 sessions of HRV-BF training. Moreover, in these patients increased HRV indices (SDNN, LF, $\ln [\mathrm{LF}]$, and total power) after HRV-BF training were observed, not only at post-testing and 1-month follow-up but also at post-training for each HRV-BF session.

These results are consistent with previous studies that applied HRV-BF and observed a reduction in depressive symptoms and an increase in HRV indices, in MDD patients. ${ }^{16-18)}$ After increasing the sample size and introducing a control group, we targeted MDD patients who 
Table 3. Psychological questionnaires scores, breathing rates, HRV indices at pre-test, post-test, and follow-up for the HRV-BF group ( $\mathrm{n}=18$ )

\begin{tabular}{|c|c|c|c|c|c|c|c|}
\hline \multirow{2}{*}{ Variable } & \multicolumn{3}{|c|}{ Time } & \multirow{2}{*}{$\mathrm{F}$} & \multirow{2}{*}{$p$} & \multirow{2}{*}{$\eta_{p}^{2}$} & \multirow{2}{*}{$\begin{array}{l}\text { Bonferroni's } \\
\text { post-hoc } \\
\text { comparison }\end{array}$} \\
\hline & Pre-test (1) & Post-test (2) & Follow-up (3) & & & & \\
\hline \multicolumn{8}{|l|}{ BDI-II } \\
\hline Total score & $24.06 \pm 13.48$ & $13.22 \pm 10.52$ & $16.94 \pm 15.14$ & $12.89^{* * *}$ & $<0.001$ & 0.43 & $1>2,3$ \\
\hline Cognitive depression & $17.06 \pm 11.84$ & $9.17 \pm 8.21$ & $11.83 \pm 12.16$ & $12.85^{* * *}$ & $<0.001$ & 0.43 & $1>2,3$ \\
\hline Somatic depression & $7.00 \pm 2.79$ & $4.06 \pm 2.69$ & $5.11 \pm 3.31$ & $7.46^{* *}$ & 0.002 & 0.31 & $1>2$ \\
\hline BAI, total score & $21.11 \pm 10.90$ & $11.72 \pm 8.10$ & $14.50 \pm 11.74$ & $12.32^{* * *}$ & $<0.001$ & 0.42 & $1>2,3$ \\
\hline \multicolumn{8}{|l|}{ PSQI } \\
\hline Total score & $12.28 \pm 3.50$ & $9.78 \pm 3.69$ & $10.39 \pm 4.18$ & $7.45^{* *}$ & 0.002 & 0.31 & $1>2$ \\
\hline Subjective sleep quality (C1) & $2.00 \pm 0.77$ & $1.39 \pm 0.70$ & $1.78 \pm 0.81$ & $5.55^{* *}$ & 0.008 & 0.25 & $1>2$ \\
\hline Sleep latency (C2) & $1.67 \pm 0.91$ & $1.56 \pm 0.86$ & $1.72 \pm 0.83$ & 0.38 & 0.690 & 0.02 & \\
\hline Sleep duration (C3) & $1.78 \pm 1.06$ & $1.22 \pm 0.94$ & $1.22 \pm 1.06$ & $3.32^{*}$ & 0.048 & 0.16 & $1>2$ \\
\hline Habitual sleep efficiency (C4) & $1.17 \pm 1.15$ & $1.06 \pm 1.06$ & $0.56 \pm 0.92$ & 2.92 & 0.084 & 0.15 & \\
\hline Sleep disturbance (C5) & $1.44 \pm 0.51$ & $1.39 \pm 0.61$ & $1.56 \pm 0.71$ & 0.62 & 0.542 & 0.04 & \\
\hline Use of sleeping medications (C6) & $2.17 \pm 1.20$ & $1.78 \pm 1.26$ & $2.00 \pm 1.37$ & 1.25 & 0.299 & 0.07 & \\
\hline Daytime dysfunction (C7) & $2.06 \pm 0.94$ & $1.39 \pm 0.85$ & $1.56 \pm 0.92$ & $5.14^{*}$ & 0.011 & 0.23 & $1>2$ \\
\hline Total time spent asleep (min) & $28.06 \pm 15.82$ & $22.22 \pm 13.53$ & $30.17 \pm 20.69$ & $3.54^{*}$ & 0.040 & 0.17 & NS \\
\hline Total sleep time (hr) & $5.58 \pm 1.59$ & $6.64 \pm 1.16$ & $6.59 \pm 1.37$ & $7.40^{* *}$ & 0.002 & 0.30 & $2,3>1$ \\
\hline Sleep efficiency (\%) & $71.72 \pm 15.43$ & $79.17 \pm 12.58$ & $86.28 \pm 14.03$ & $13.16^{* * *}$ & $<0.001$ & 0.44 & $3>1,2$ \\
\hline \multicolumn{8}{|l|}{ PSAS } \\
\hline Total score & $43.11 \pm 13.70$ & $33.83 \pm 12.09$ & $38.06 \pm 14.02$ & $8.35^{* *}$ & 0.001 & 0.33 & $1>2$ \\
\hline Cognition arousal & $24.89 \pm 9.07$ & $18.56 \pm 7.88$ & $22.11 \pm 9.52$ & $7.92^{* *}$ & 0.002 & 0.32 & $1,3>2$ \\
\hline Physical arousal & $18.22 \pm 6.37$ & $15.28 \pm 5.54$ & $15.94 \pm 5.85$ & $3.31^{*}$ & 0.049 & 0.16 & \\
\hline Breathing rate (breath/min) & $14.56 \pm 4.36$ & $11.02 \pm 4.06$ & $11.07 \pm 3.63$ & $6.44^{* *}$ & 0.004 & 0.26 & $1>2,3$ \\
\hline \multicolumn{8}{|l|}{ HRV indices } \\
\hline SDNN (ms) & $39.80 \pm 16.71$ & $49.97 \pm 26.92$ & $51.94 \pm 25.26$ & $6.57^{* *}$ & 0.004 & 0.28 & $2,3>1$ \\
\hline $\operatorname{LF}\left(\mathrm{ms}^{2}\right)$ & $158.42 \pm 233.18$ & $583.46 \pm 932.19$ & $676.78 \pm 1058.31$ & $5.03 *$ & 0.012 & 0.23 & \\
\hline $\mathrm{HF}\left(\mathrm{ms}^{2}\right)$ & $147.89 \pm 114.23$ & $185.08 \pm 182.59$ & $184.85 \pm 163.03$ & 0.72 & 0.497 & 0.04 & \\
\hline $\ln (\mathrm{LF})\left(\mathrm{ms}^{2}\right)$ & $4.40 \pm 1.23$ & $5.40 \pm 1.47$ & $5.64 \pm 1.38$ & $10.62^{* * *}$ & $<0.001$ & 0.38 & $2,3>1$ \\
\hline $\ln (\mathrm{HF})\left(\mathrm{ms}^{2}\right)$ & $4.55 \pm 1.22$ & $4.59 \pm 1.28$ & $4.75 \pm 1.10$ & 0.33 & 0.724 & 0.02 & \\
\hline LF/HF ratio & $1.25 \pm 1.39$ & $6.83 \pm 11.04$ & $5.29 \pm 6.66$ & 2.75 & 0.098 & 0.14 & \\
\hline Total power $\left(\mathrm{ms}^{2}\right)$ & $596.80 \pm 573.68$ & $1,052.27 \pm 1,131.51$ & $1,098.48 \pm 1,156.35$ & $5.18^{*}$ & 0.011 & 0.23 & $2>1$ \\
\hline
\end{tabular}

Values are presented as mean \pm standard deviation.

HRV, heart rate variability; BF, biofeedback; BDI-II, Beck Depression Inventory-II; BAI, Beck Anxiety Inventory; PSQI, Pittsburgh Sleep Quality Index; NS, not significant; PSAS, Pre-Sleep Arousal Scale; SDNN, standard deviation of normal to normal intervals; LF, low frequency; HF, high frequency.

${ }^{*} p<0.05,{ }^{* *} p<0.01,{ }^{* * *} p<0.001$.

present with comorbid insomnia. Regarding the effects of HRV-BF on psychological aspects, this study showed that HRV-BF training reduced the occurrence of depression and anxiety, and alleviated symptoms of insomnia. The possible explanation for the effect of HRV-BF on emotional regulation was that the vagal afferent pathway to the brain areas, such as the amygdala and orbitofrontal cortex, affects emotional regulation process. ${ }^{29)}$ Therefore, decreased depression and anxiety after HRV-BF was found in patients with MDD.

The improvements on insomnia symptoms were observed from both BDI-II and PSQI in this study, however, the insomnia constructs may be varied between these two psychological questionnaires. The BDI-II only assessed the change in the sleeping pattern within a two-week period; we did not know the quantity and direction of sleep changes. The insomnia symptoms from PSQI not only assess total score of sleep quality but also the component score of $\mathrm{C} 1, \mathrm{C} 3$, and C7. Therefore, the improvements on insomnia symptoms by HRV-BF training were acquired in this study, included improved subjective sleep quality, sleep duration (total sleep time), daytime function, and sleep efficiency; as well as decreased total time spent asleep and pre-sleep arousal. Even though cortisol hyperarousal has been associated with primary insomnia, ${ }^{30)}$ very few studies have explored the effect of HRV-BF in patients with MDD and insomnia comorbidity. HRV-BF training is used to regulate and restore the hyperactivation 

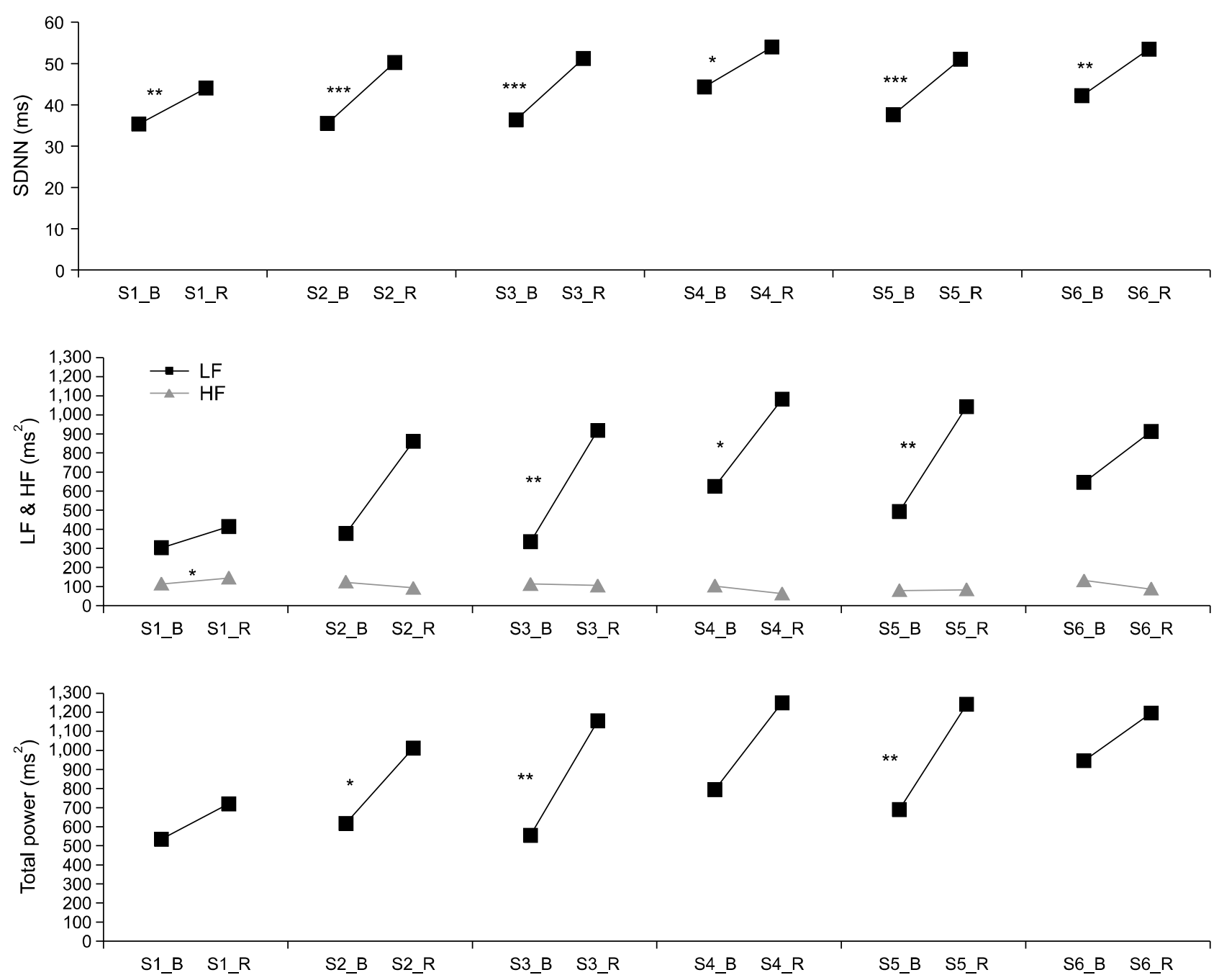

Fig. 2. Differences on heart rate variability (HRV) indices between pre- and post-trainings during each HRV biofeedback session. $\mathrm{LF}$, low-frequency power; HF, high-frequency power; SDNN, standard deviation of normal to normal intervals. ${ }^{*} p<0.05,{ }^{* *} p<0.01$.

of the ANS, and treatment effectiveness has already been reported in physical illness and mental disorder. ${ }^{29)}$

Regarding the effects of HRV-BF on physical aspects, some of the articles have identified the treatment index of HRV, such as increased SDNN, rMSSD, and total power. ${ }^{14,29,31)}$ Our data indicated that HRV-BF training increases HRV indices (SDNN, LF, and total power) and is consistent with Karavidas et al. ${ }^{16)}$ who similarly detected a significantly improved SDNN at training session 4. Nevertheless, this effect on HRV indices was not maintained to post-testing or at follow-up in their study. In contrast, our data indicate that HRV indices (including SDNN, LF, and total power) were increased not only at post-testing but also at 1-month follow-up and during
HRV-BF training sessions. The physiological mechanisms of increasing SDNN and total power involves the increase of total variability among cardiac function and vagal activation, improvement of homeostasis in the baroreceptor or baroreflex sensitivity, autonomic regulation, and stimulation of the vagal efferent system. ${ }^{14,29,31)}$ In this study, participants were trained to slow-down their breathing rates at a resonance frequency, about 5.5 to 6 breaths/minutes (approximately 0.09 to $0.1 \mathrm{~Hz}$ ). The phase relationships between HR oscillations and breathing at resonance frequencies is that maximizing HR amplitude and baroreflex activity result in an increased HRV. Therefore, participants significantly increased their SDNN, LF, and total power at each HRV-BF training session, and at post-test- 
ing after HRV-BF.

Nevertheless, the physiological mechanisms behind the increase of LF after HRV-BF remain unclear. Some studies have suggested that LF is a baroreflex gain index while breaths at about 6 breathes/per minute, which modulates HR and blood pressure through changes in vagal efferent activity. ${ }^{14,32-35)}$ Vagal activity regulates the sinoatrial node, which influences HR, blood pressure, and cardiac output, and leads to increased LF. When vagal activity was withdrawn, HRV indices decreased. ${ }^{14,32-34)}$ Therefore, HRV-BF intervention activates the efferent vagal activity that increases $\log \mathrm{LF}$ and regulates baroreflex gain. ${ }^{29,35-37)}$

The beneficial effects of HRV-BF involve increasing baroreflex and vagal modulation of the autonomic outflow $^{32-35)}$ thus, restoring the balance between sympathetic and parasympathetic activation and maximizing $\mathrm{HR}$ amplitude by slow and deep diaphragmatic breathing. Therefore, increasing SDNN and total power of HRV were found after HRV-BF. In the present study, we observed increased SDNN, LF, and total power after HRV-BF, supporting the arguments mentioned above. Finally, there is an increasing number of patients that are subjected to training on how to slow down and stabilize their breathing rates, as a form of relaxation. ${ }^{14,27)}$

The study has several limitations. First, although this study extrapolated the indices of autonomic activations from a 5-minute HRV to reflex the indices of autonomic activations, this study did not perform 24-hour or pre-sleep ECGs, so as to extrapolate additional data for HRV indices. The changes in HRV indices prior to sleep are still unknown. Second, MDD is a heterogeneous disease, it may comorbidity with anxiety disorder or specifier with anxious distress or melancholia. ${ }^{5-8)}$ This study did not assess the differences between MDD comorbidities or specifiers on HRV indices.

Third, although we only included MDD patients with a PSQI higher than 6 in the HRV-BF and control groups, we did not compare HRV indices between MDD patients with or without insomnia comorbidity, among the cohort populations. Therefore, we do not know if HRV is a suitable index for autonomic activation. Finally, there was no significant difference on medications used at pre-testing between two groups; however, this study did not control for medications and did not require the participants to be medication-free prior to the breathing measures and ECG recordings, the medications may influence the observed results. Moreover, this study did not acquire the medications recorders at post-testing and 1-month follow-up, the changes of medications after HRV-BF training are still unknown.

In conclusion, 6 sessions of HRV-BF training improved autonomic balance and baroreflex and decreased symptoms of depression and insomnia among patients with MDD and comorbid insomnia. HRV-BF training together with psychosocial intervention, will likely be considered in the future as standard clinical practice, for improving both the physical and psychological aspects of patients with the mental and physical illness.

\section{Acknowledgments}

This study was funded by Kaohsiung Medical University, Taiwan, and grant number: KMU-Q110013.

We would like to thank the student assistants Miss Hui Ping Chan for data collection.

\section{REFERENCES}

1. World Health Organization (WHO). Global burden of mental disorders and the need for a comprehensive, coordinated response from health and social sectors at the country level [Internet]. Geneva: WHO; 2012 [cited at 2018 Feb 6]. Available from: http://apps. who.int/iris/handle/10665/78898? locale=en

2. Sunderajan P, Gaynes BN, Wisniewski SR, Miyahara S, Fava $\mathrm{M}$, Akingbala $\mathrm{F}$, et al. Insomnia in patients with depression: a STAR*D report. CNS Spectr 2010;15:394-404.

3. Sgoifo A, Carnevali L, Alfonso Mde L, Amore M. Autonomic dysfunction and heart rate variability in depression. Stress 2015; 18:343-352.

4. Maes J, Verbraecken J, Willemen M, De Volder I, van Gastel A, Michiels N, et al. Sleep misperception, EEG characteristics and autonomic nervous system activity in primary insomnia: a retrospective study on polysomnographic data. Int J Psychophysiol 2014;91:163-171.

5. Kemp AH, Quintana DS, Felmingham KL, Matthews S, Jelinek HF. Depression, comorbid anxiety disorders, and heart rate variability in physically healthy, unmedicated patients: implications for cardiovascular risk. PLoS One 2012;7:e30777.

6. Kemp AH, Quintana DS, Gray MA, Felmingham KL, Brown K, Gatt JM. Impact of depression and antidepressant treatment on heart rate variability: a review and meta-analysis. Biol Psychiatry 2010;67:1067-1074.

7. Kemp AH, Quintana DS, Gray MA. Is heart rate variability reduced in depression without cardiovascular disease? Biol Psychiatry 2011;69:e3-e4.

8. Kemp AH, Quintana DS, Quinn CR, Hopkinson P, Harris AW. Major depressive disorder with melancholia displays robust 
alterations in resting state heart rate and its variability: implications for future morbidity and mortality. Front Psychol 2014:5:1387.

9. Ha JH, Park S, Yoon D, Kim B. Short-term heart rate variability in older patients with newly diagnosed depression. Psychiatry Res 2015;226:484-488.

10. Kemp AH, Brunoni AR, Santos IS, Nunes MA, Dantas EM, Carvalho de Figueiredo R, et al. Effects of depression, anxiety, comorbidity, and antidepressants on resting-state heart rate and its variability: an ELSA-Brasil cohort baseline study. Am J Psychiatry 2014;171:1328-1334.

11. Wang Y, Zhao X, O'Neil A, Turner A, Liu X, Berk M. Altered cardiac autonomic nervous function in depression. BMC Psychiatry 2013;13:187.

12. Spiegelhalder K, Fuchs L, Ladwig J, Kyle SD, Nissen C, Voderholzer $\mathrm{U}$, et al. Heart rate and heart rate variability in subjectively reported insomnia. I Sleep Res 2011;20: 137-145.

13. Pigott HE, Leventhal AM, Alter GS, Boren JJ. Efficacy and effectiveness of antidepressants: current status of research. Psychother Psychosom 2010;79:267-279.

14. Lehrer PM, Gevirtz R. Heart rate variability biofeedback: how and why does it work? Front Psychol 2014,5:756.

15. Lehrer PM, Vaschillo E, Vaschillo B. Resonant frequency biofeedback training to increase cardiac variability: rationale and manual for training. Appl Psychophysiol Biofeedback 2000;25:177-191.

16. Karavidas MK, Lehrer PM, Vaschillo E, Vaschillo B, Marin H, Buyske S, et al. Preliminary results of an open label study of heart rate variability biofeedback for the treatment of major depression. Appl Psychophysiol Biofeedback 2007;32:19-30.

17. Siepmann M, Aykac V, Unterdörfer J, Petrowski K, Mueck-Weymann M. A pilot study on the effects of heart rate variability biofeedback in patients with depression and in healthy subjects. Appl Psychophysiol Biofeedback 2008;33: 195-201.

18. Caldwell YT, Steffen PR. Adding HRV biofeedback to psychotherapy increases heart rate variability and improves the treatment of major depressive disorder. Int I Psychophysiol 2018;131:96-101.

19. American Psychiatric Association. Diagnostic and statistical manual of mental disorders, 5th ed (DSM-5). Washington, DC:American Psychiatric Publishing;2013.

20. Tsai PS, Wang SY, Wang MY, Su CT, Yang TT, Huang CJ, et al. Psychometric evaluation of the Chinese version of the Pittsburgh Sleep Quality Index (CPSQI) in primary insomnia and control subjects. Qual Life Res 2005;14:1943-1952.

21. Lu ML, Che HH, Chang SW, Shen WW. Reliability and validity of the Chinese version of the Beck Depression Inventory-II. Taiwanese J Psychiatry 2002;16:301-310.
22. Storch EA, Roberti JW, Roth DA. Factor structure, concurrent validity, and internal consistency of the Beck Depression Inventory-second edition in a sample of college students. Depress Anxiety 2004;19:187-189.

23. Beck AT, Steer RA. Manual for the Beck anxiety inventory. San Antonio, TX:Psychological Corporation; 1990.

24. Che HH, Lu ML, Chen HC, Chang SW, Lee YJ. Validation of the Chinese version of the Beck Anxiety Inventory. Formosan J Med 2006; 10:447-454.

25. Nicassio PM, Mendlowitz DR, Fussell JJ, Petras L. The phenomenology of the pre-sleep state: the development of the pre-sleep arousal scale. Behav Res Ther 1985;23:263-271.

26. Jan YW, Chen CW, Yang CM, Lin SC. Validation of the Chinese version of the Pre-Sleep Arousal Scale (PSAS). Arch Clin Psycho 2009;4:51-58.

27. Lin IM, Fan SY, Lu HC, Lin TH, Chu CS, Kuo HF, et al. Randomized controlled trial of heart rate variability biofeedback in cardiac autonomic and hostility among patients with coronary artery disease. Behav Res Ther 2015;70:38-46.

28. Stevens JC. Applied multivariate statistics for the social sciences. Hillsdale, NJ:Lawrence Erlbaum Associates;2002.

29. Wheat AL, Larkin KT. Biofeedback of heart rate variability and related physiology: a critical review. Appl Psychophysiol Biofeedback 2010;35:229-242.

30. Bonnet MH, Arand DL. Hyperarousal and insomnia: state of the science. Sleep Med Rev 2010;14:9-15.

31. Moss D, Shaffer F. The application of heart rate variability biofeedback to medical and mental health disorders. Biofeedback 2017;45:2-8.

32. Reyes del Paso GA, Langewitz W, Mulder LJ, van Roon A, Duschek S. The utility of low frequency heart rate variability as an index of sympathetic cardiac tone: a review with emphasis on a reanalysis of previous studies. Psychophysiology 2013;50:477-487.

33. Shaffer F, McCraty R, Zerr CL. A healthy heart is not a metronome: an integrative review of the heart's anatomy and heart rate variability. Front Psychol 2014;5:1040.

34. Shaffer F, Ginsberg JP. An overview of heart rate variability metrics and norms. Front Public Health 2017;5:258.

35. Lehrer $P$, Vaschillo $E$, Trost Z, France CR. Effects of rhythmical muscle tension at $0.1 \mathrm{~Hz}$ on cardiovascular resonance and the baroreflex. Biol Psychol 2009;81:24-30.

36. Billman GE. The effect of heart rate on the heart rate variability response to autonomic interventions. Front Physiol 2013;4: 222.

37. Lehrer PM, Vaschillo E, Vaschillo B, Lu SE, Eckberg DL, Edelberg R, et al. Heart rate variability biofeedback increases baroreflex gain and peak expiratory flow. Psychosom Med 2003;65:796-805. 